\section{The easy way to successful regeneration}

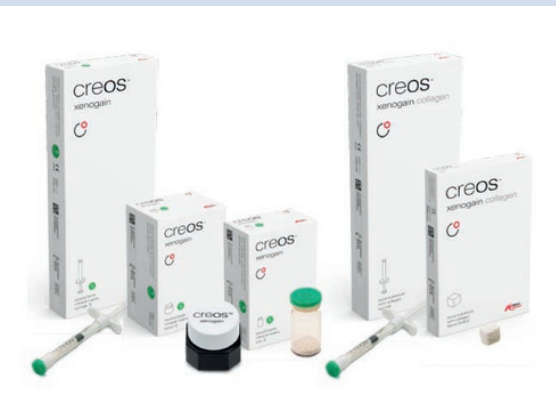

creos xenogain from Nobel Biocare.

Easy to handle and able to provide quick, yet precise applications, creos xenogain is available in multiple granule sizes and volume options to meet the needs of the clinician.

A biocompatible solution that has a calcium phosphate ratio similar to human bone, creos xenogain has been proven to promote new bone formation in the oral cavity, says the company. By acting as a slowly resorbing scaffold in implant procedures, it maintains space for bone generation and integrates with the new bone as it forms, building the solid foundation necessary for success.

For more information, call Nobel Biocare on 02087563300 or visit www.nobelbiocare.com/uk/en/creos.

\section{Network, learn and have fun at oral health conference}

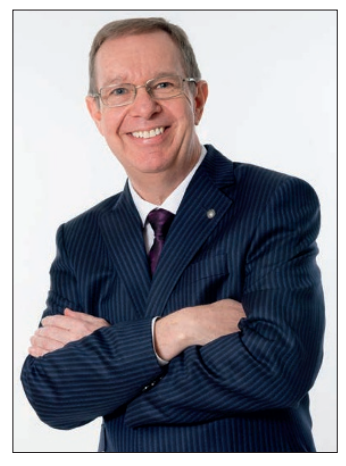

The British Society of Dental Hygiene and Therapy (BSDHT) is keen to promote this year's Oral Health Conference and Exhibition (OHC) entitled 'The future is yours', taking place at the Telford International Centre, Shropshire, on 23-24 November 2018.

The conference and exhibition will provide an array of learning and networking opportunities for all UK dental hygienists and dental therapists.

Tim Ives will be one of the many not-tobe-missed speakers, presenting a session on 'LipZip - Incorporating Buteyko breathing and oral myology into practice and beyond' on 23 November 2018.

Ives said: 'During my lecture, I will examine the importance of correct breathing, covering how to do it, how to identify mouth breathers and how to help them become nose breathers.

'This is important for dental hygienists and dental therapists because we should be providing holistic care and there is a direct effect on the oral cavity for patients who are breathing incorrectly. For example, it increases the risk of disease and it can also have an impact on growth of the jaws in children.

'OHC 2018 provides the ideal opportunity to network, learn and have some fun so I would definitely encourage all dental hygienists and dental therapists to attend.'

For more information, please visit www.bsdht.org.uk/OHC2018 or call 01788575050 or email enquiries@bsdht.org.uk.

\section{Minimise your mercury output}

It is estimated that $100 \mathrm{~kg}$ of mercury is deposited into our environment from waste amalgam in dental practices every year, which is having serious effects on the environment and may be having an impact on general health as well. It is time for dental practices to take action.

Amalgam separators from Initial Medical are the ideal way to limit the amount of mercury that enters the wastewater from dental practices. Using the principle of sedimentation, these separators remove up to $99.8 \%$ of amalgam particles from wastewater streams.

Able to be placed on all routes whereby wastewater could reach the main sewage system including sinks where instruments are washed during treatment, these separators also contain no moving parts or electronics, providing noiseless function and minimal surgery downtime during services.

More information is available by visiting www.initial.co.uk/ medical or by calling 08708504045 .

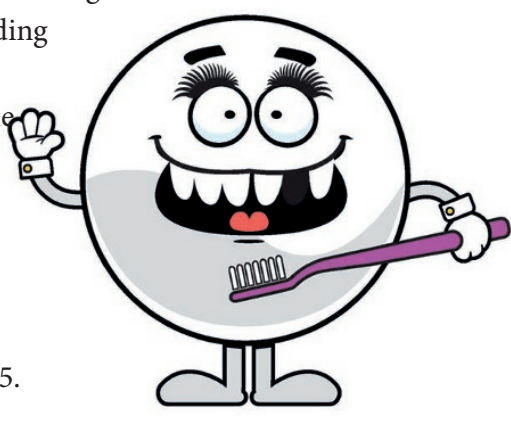

\section{Pain-less prevention}

Toothache, or oral pain, can impact dramatically on a patient's quality of life and many patients choose to administer pain relief at home in the form of paracetamol, rather than see a dentist.

However, this can lead to accidental overdose.

The only way to avoid dental pain is to employ good preventive care between appointments. The TANDEX range of brushes, interdental and interspace brushes, plus adjunctive products, is everything a patient needs to keep their mouth healthy.

Recommended by dentists, dental hygienists and dental therapists, TANDEX products are high quality, robust and easy to use, says the company. TANDEX understands that prevention is the key to maintaining oral health, so every patient must be given the right tools to do this properly.

The designs are stylish and functional. For patients who have not yet experienced the benefits of interdental cleaning, the FLEXI will convert them immediately. PREVENT WASH and PREVENT FRESH are mouthwashes that will strengthen the enamel and have an antibacterial effect while PREVENT FRESH will give fresh breath too.

For more information on Tandex's range of products, visit www.tandex.dk.

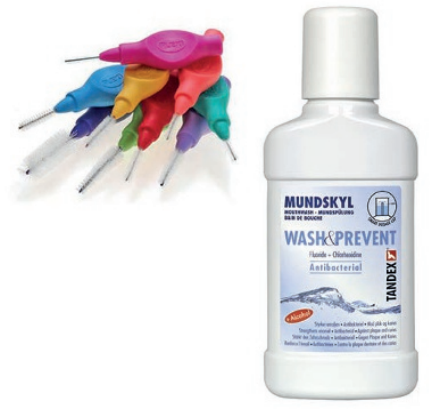

